\title{
Interaction with a TV Companion App as Synopsis and Supplement
}

\author{
John Dowell \\ University College London \\ London, UK \\ j.dowell@ucl.ac.uk
}

\begin{abstract}
Television companion apps on tablets and smartphones provide interactive content synchronized with TV shows. A key design question raised by this novel, multi-display, multimedia interface is whether the app's role is to be a synopsis of the show or a supplement. In other words, should the app help viewers better follow what they are watching on $\mathrm{TV}$, or offer additional enriching content to respond to interest created by the show? We developed a companion app for a documentary with both synoptic and supplementary content. A laboratory study with 28 participants examined the effect of these different types of content on the experience of using the companion and the effect on engagement with the show in terms of participants' recall. Engagement with the show was not affected by supplementary content in the app but coordinated viewing of both screens was more difficult. Design guidelines evident from these results are discussed.
\end{abstract}

\section{Author Keywords}

Second screen; companion apps; mobile devices; engagement; television.

\section{ACM Classification Keywords}

H.5.2. User Interfaces.

\section{INTRODUCTION}

Companion apps (CAs) on tablets and smartphones augment viewing of a TV show being watched on a typically larger screen $[4,13]$. They embody one of the many relationships between TV and the web made possible by the convergence of these two environments [11]. Examples of these apps have appeared for a variety of show genres including news, natural history, quiz and sport events shows. TV with CA also presents a novel multiscreen multimedia use case for researchers.

In studies of users' experiences of CAs, a dichotomy has become apparent. While some users express a preference for additional information and activity with a CA $[6,10]$, others

Permission to make digital or hard copies of all or part of this work for personal or classroom use is granted without fee provided that copies are not made or distributed for profit or commercial advantage and that copies bear this notice and the full citation on the first page. Copyrights for components of this work owned by others than ACM must be honored. Abstracting with credit is permitted. To copy otherwise, or republish, to post on servers or to redistribute to lists, requires prior specific permission and/or a fee. Request permissions from Permissions@acm.org.

CHI 2017, May 06-11, 2017, Denver, CO, USA

(C) 2017 ACM. ISBN 978-1-4503-4655-9/17/05 ..\$15.00

DOI: http://dx.doi.org/10.1145/3025453.3025459

\author{
Edward Anstead \\ University College London \\ London, UK \\ e.anstead@ucl.ac.uk
}

report being distracted from the show [1, 2, 10]. This distraction is consistent with studies of media multi-tasking that typically find a simultaneous activity produces a worse performance in a primary activity, for example when using social media in school lessons [12, 15]. Nevertheless, the potential for a $\mathrm{CA}$ to improve engagement with a factual show in terms of recall of the show content has recently been demonstrated [6].

CA design clearly needs to take account of how users will coordinate their interaction with viewing the TV. Both the content and its presentation need to be designed to create interactions that enhance rather than detract from watching the TV show [5]. For example, presentation in terms of visual complexity has been shown to affect the duration of 'looks' at a CA [14]. The study we are reporting examines the impact of content type on interactions with the CA whilst watching the show, and specifically the effects of synoptic and supplementary content supporting a science documentary.

Whether CAs should be a synopsis or supplement is a fundamental issue for their use with factual TV shows. In the pilot trial of a CA for a natural history show [10], participants said they most valued supplementary content as a "natural extension to the moment" [10]. Supplementary content may well improve viewer engagement with a TV show through active, individual exploration. However, synoptic content provides a durable representation that can reinforce the essential transience of televisual media; this may help viewers to better follow more complex, information-rich TV shows such as science documentaries or long-arc narratives [13]. So within the constraints of the metre of the TV show and the viewer's ability to process information from two displays, the CA designer must decide on the most desirable content to include, when synoptic information should be foregrounded and whether supplementary information will aid viewer engagement.

Given the existing evidence that a CA can enhance engagement with a TV show $[6,10]$, we examine the impact of the types of content provided by a CA. Specifically, we compare the effects of synoptic and supplementary CA content on (i) users' experience of watching TV with a CA; (ii) the way users divide their attention across screens, and; (iii) users' engagement with the TV show in terms of their ability to recall and understand it. 


\section{APP DESIGN AND METHOD}

\section{Show Selection and the SAOS app}

A BBC science documentary, "The Seven Ages of Starlight" [9] was chosen as the source material for our study. The show describes the stages in the lifecycle of a star, from its formation in clouds of dust and gas through to its violent death as a supernova or black hole. The show consists of separate chapters, each dealing with a particular stage that can be watched separately. The chapter concerning supernovae was chosen on account of its balance of factual and conceptual content. At some 11 minutes long, the chapter allowed participants to become immersed during the study. 18 multiple-choice style 'probe' questions (ProgQs) about the supernova chapter were created including, for example, the following: 'The largest stars are as much as 200 times the mass of the sun? (True/False)'; 'The elements making up the earth and the elements making up the stars are: (a) Exactly the same; (b) Totally different; (c) Some the same, some different; (d) I don't know'.

We constructed a prototype CA for this show: the Seven Ages of Starlight (SAOS) app. It reflects design elements from exemplar CAs created by both researchers [13] and developers [10]. Two versions of the SAOS app were constructed, a baseline 'synoptic' version and a 'supplementary' version providing additional supplementary information. On launching either version, the show video begins playback on the TV display. As the show progresses, navigational thumbnail buttons appear across the top of the SAOS app (figure 1) offering the viewer the option of a new page of companion information synchronised with the show. In this way the viewer is able to manage the synchrony between displays and coordinate their viewing. They monitor the app for the appearance of new thumbnails and access the linked page at a moment of their choosing.

Each CA page is a synopsis of the concepts and facts contained in the show during a particular epoch. This content is presented as a series of bullet points. However, in the supplementary version of the application, some of the bullet points are folding 'twisty' menus (visible as $\boldsymbol{\wedge}$ in Figure 1); tapping one reveals a further indented, bulleted set of supplementary information related to the content of the show

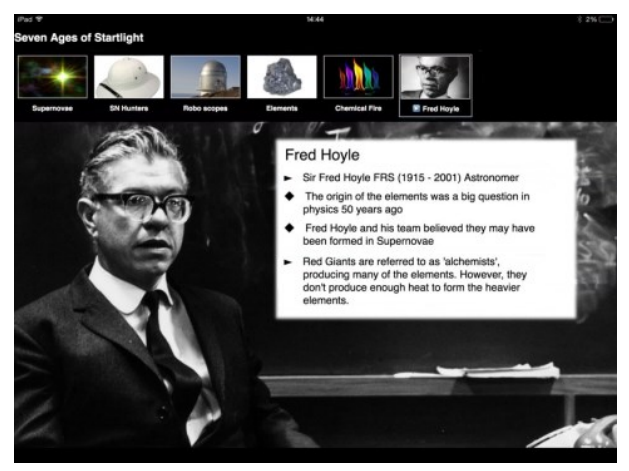

Figure 1. The SAOS app, a companion application developed for the Seven Ages of Starlight TV documentary [9]. but taking the concepts further and adding details or background.

\section{Method}

The study design was a between subjects comparison of the synopsis and supplementary versions of the SAOS app. The synopsis group used the SAOS app with only synoptic content, i.e., content contained in the show; the supplementary group used the app version containing synoptic and supplementary information. The two groups were compared through their responses to probe and survey questions.

Recall of the TV show content was measured directly after viewing with the ProgQ probe questions. An additional 12 multi-choice probe questions (SupQs) were used to assess recall and understanding of the supplementary app content for participants in the supplementary group. As participants could choose whether to see each element of supplementary information, any SupQ was only asked if the participant had opened the associated twisty menu on the application.

Participants' experiences of using the SAOS app were elicited with a questionnaire asking about their appreciation of the app, their experience of using it including how it changed their TV viewing and how they managed their visual attention over both displays:

EQ1: How likely are you to use a CA in the future while watching a TV programme: 5-point scale from "Very likely" to "Very unlikely"

EQ2: How much did you enjoy using the application while watching? 5-Point Likert

EQ3: Do you think using the app helps you to recall the content of programmes such as this? 5-Point Likert

EQ4: What did you think about the quantity of facts and information that was contained in the app? 5-point scale from "Much too little information" to "Much too much information"

EQ5: Do you think that the content of the app should reiterate what you are watching or should it provide additional content not in the programme? Multiple choice responses; "Reinforce what is on the television"; "Provide extra information only"; "A combination of the two"; "None of the above"

EQ6: While watching the programme did you find it difficult to know which device to look at? 5-point scale from "Very difficult" to "Very easy"

EQ7: How easy did you find watching the television and reading content on the tablet at the same time? 5-point scale from "Very difficult" to "Very easy"

EQ8: Did you try and read the content on the app at the same time as it was on the television? Yes/No

EQ9: How much of the application's content did you read? 5-point scale from "All or most of the content" to "Little or none of the content"

EQ10: Do you feel you had enough time to read the content you wanted to? Yes/No 
28 participants were recruited for the study, 15 male and 13 female, with an approximate average age of 26 years. Recruitment of participants excluded any who had taken a physics qualification beyond age 16. Each participant's prior understanding of astronomy was assessed with 8 multichoice questions at secondary education level; these revealed no significant difference between individuals. Each participant was then introduced to the SAOS app and its features were explained to them, including the twisty menus in the supplementary version. Participants then began watching the supernovae chapter of the show using the app. They watched uninterrupted without pausing or rewinding and then answered the probe questions before finally completing the questionnaire. Two probe question sets were produced, one for each group with the ProgQ questions common to both groups. The SupQ probe questions were individually selected to correspond to the supplementary content a participant had chosen to see by opening a twisty menu in the SAOS app.

\section{RESULTS}

We first examine the user experience of this multiscreen multimedia interface and how it is affected by adding supplementary information to the CA. We then examine how adding the supplementary information modified the way users divided their attention between the two screens. Finally, we examine the effect on engagement with the TV show of adding supplementary information to the CA.

\section{Experience of using the multi display multimedia interface}

Participants reflected on the experience of using the app through several survey questions.

(EQ1). If they were given the choice of using a CA like the SAOS app, the majority of participants said that they would use it $\left(\chi^{2}(4)=12.357, p=.015\right)$. A Mann-Whitney test found no difference in response between the two groups ( $U=67.7$, $\mathrm{Z}=1.477, \mathrm{p}=.164)$. So the addition of the supplementary information was not found to increase or decrease participants' preference for using an app like this in the future.

(EQ2). Watching the TV using the SAOS app was enjoyable for most participants $\left(\chi^{2}(4)=11.643, p=.020\right)$ and the synoptic and supplementary version groups did not differ in their responses to this question $(U=62.0, \mathrm{Z}=1.759, \mathrm{p}=.104)$. So the addition of the supplementary information to the SAOS app was not found to increase or decrease participants' enjoyment of using it.

(EQ3). The SAOS app helps recall of the show in the opinion of most participants $\left(\chi^{2}(4)=16.643, p=.002\right)$. A borderline difference was found in the ratings of the two groups $(U=$ $56.5, \mathrm{Z}=2.133, \mathrm{p}=.056$ ). The addition of the supplementary information may lead the supplementary version group to believe that the app will be less helpful in recalling the show they have watched.
(EQ4). The quantity of facts and other information contained in the app was judged to be about right by most participants $\left(\chi^{2}(2)=7.357, p=.025\right)$. The two groups did not differ in their ratings ( $U=93.0, Z=.254, p=.839$ ). Since the majority of participants in the supplementary group chose to look at the majority of the supplementary information items, it is clear that this additional information was not experienced as unwelcome or excessive.

(EQ5). CAs like the SAOS app should both repeat the content of the TV show and extend that content, in the opinion of most participants $\left(\chi^{2}(3)=32.857, \quad \mathrm{p}=.001\right)$. Preferences for the kind of content in the CA were not correlated with the two forms of the app $(\chi(28)=3.607$, $\mathrm{p}=.307)$. Five of the supplementary version participants would prefer an app that only contained supplementary information whilst only one of the synopsis group had that preference. Almost none of the participants in either group would prefer the app to only contain synoptic information. Although the synopsis group had not expressed a general dissatisfaction with their version of the app, it is clearly implied that they saw the opportunity for the app to extend beyond echoing the content of the show.

\section{Dividing their attention}

The experience of dividing attention between the two displays was reported in several of the survey questions.

(EQ6). Knowing when to look at which screen was reported as difficult by most participants $\left(\chi^{2}(3)=18.000, \mathrm{p}=.000\right)$. There was also a borderline significant difference between the two groups on this question $(U=56.5, \mathrm{Z}=2.133, \mathrm{p}=.056)$. The supplementary information appeared to make it marginally harder to choose when to look at the app and when at the TV screen.

(EQ7). The ease of reading the app content and listening to the TV at the same time was rated significantly differently by the two groups ( $U=42.0, Z=2.710, p=.009$ ). So the addition of supplementary content made it harder to read visual text on the app and listen to the TV show at the same time.

(EQ8). Most participants reported trying to synchronise their reading of text on the app with listening to the show $\left(\chi^{2}(1)=11.571, p=.001\right)$. The synoptic content that both groups accessed could be read in synchrony with the TV, even though the visual text was not a transcription of the spoken content. No correlation was found between use of the different versions of the app and attempting to synchronise reading and listening $(\chi(1)=2.19, \mathrm{p}=139)$. The baseline mode of most participants then was to try to coordinate reading and listening and this was unchanged by the availability of the supplementary information.

(EQ9). The majority of participants believed that they had read most of the content on the app $\left(\chi^{2}(2)=15.071, \mathrm{p}=.001\right)$. This question was intended to elicit any sense of having been overwhelmed by the amount of content in the app and whether a deliberate choice had been made to not read all the content. No significant difference was found between the 
groups $(U=82.0, Z=.926, \mathrm{p}=.482)$, in spite of the greater amount of content seen by the supplementary version group.

(EQ10). Most of the synopsis group thought there was enough time to read the content of the CA, in contrast with the supplementary group $(\chi(1)=9.14, p=.002)$. The addition of the supplementary information clearly lies behind this difference and even though participants could choose whether to see each of the seven items of supplementary information, and some chose not to see some items, the overall sense it created for the participants was of there being insufficient time to view the information they were interested in seeing.

\section{Engagement with the TV show}

Engagement with the TV show was not affected by the addition of supplementary information. A t-test found no significant difference in the proportion of correct answers to the ProgQs $(\mathrm{T}(26)=-.002, \mathrm{p}=.998)$ between the synopsis (71.8, sd=7.71) and supplementary (71.8, $\mathrm{sd=7.69)}$ groups.

A significant difference was found $(\mathrm{T}(26)=3.51, \mathrm{p}=.004)$ in a paired samples comparison of the supplementary groups' answers (71.8, sd=7.69) and SupQs (51.3, sd=22.29). The difference indicates a worse understanding and recall of content that was only present in the app. Further analysis of the supplementary groups' use of the supplementary information showed that most participants chose to look at most of the supplementary information. However, the number of SupQs this group correctly answered did not correlate with the number of supplementary items they had viewed, $(\mathrm{r}=-.372, \mathrm{p}=.173)$; in other words, opening more items of supplementary information didn't improve their score in answering questions about those items.

\section{DISCUSSION AND IMPLICATIONS}

Whether CAs should provide only a synopsis or additional supplementary content is a key design issue for their use with factual TV shows. Adding supplementary content to the SAOS app did not affect the experience of watching the show but it did affect how participants used the app and in particular, the reported difficulty of sharing attention over both displays. The supplementary group found it harder to read the content of the app and to listen to the TV show at the same time, although they still attempted to coordinate their reading of the app content with listening to the TV. Knowing when to look at each display was hard for all participants and marginally harder for the supplementary group.

Recall of the show, a measure of viewer engagement, was not affected by the addition of supplementary content to the app. However the supplementary content was itself recalled less well than the synoptic show content. Although most participants chose to look at the supplementary content, participants appear not to have read it or processed it sufficiently. The difficulty of absorbing supplementary information whilst watching the TV show is evident.
The study also revealed how participants adapted to using the app, particularly in how they shared their attention between the displays. All participants reported difficulty with knowing where to look when using the app. Participants were divided however on whether it was difficult to read the content of the app and listen to the TV show at the same time. Most participants said they attempted to coordinate their reading and listening, supporting other observations of users making many short coordinated 'looks' at CAs [3, 6, $8]$.

Users' views about the purpose and design of CAs for factual TV were also revealed. The quantity of facts and other information contained in the SAOS app was judged to be suitable and participants reported having enough time to read the text content of the app. They believed that CAs should both repeat the content of the TV show and provide additional information. Our participants enjoyed using the SAOS app and said that given the choice they would use such an app in the future. They believed the app helped them to engage with the TV show.

These results obtained with our SAOS app would be expected to be replicated with other CAs developed for information rich and factual TV shows. TV shows vary greatly and users' interactions with CAs have been found to differ systematically by show genre [7]. Many of our results may therefore not apply to CAs for other TV show genres; moreover, the distinction between synoptic and supplementary content may have limited meaning for other genres of TV show. However the preference we found for using a CA has also been found in studies with other genres of TV show $[10,11]$.

Design guidelines for CAs for factual TV shows follow from our results. Both synoptic and supplementary content should be provided by CAs accompanying factual shows. CAs should present no more than a moderately sized paragraph of supplementary content and any larger texts should be accessible only after watching the show. CAs should help viewers coordinate their gaze shifts and 'knowing where to look', for example by making new content available during natural transitions in the show's narrative. CAs should help viewers to coordinate their reading of companion content by giving them control over the display and choice over when to display synchronised companion content, rather than simply 'pushing' content at them; for example, thumbnails can cue the availability of new content pages and folding menus offer access to supplementary text. CAs should also support users who want to coordinate their reading with listening to the show, for example by visually indicating place in displayed text that corresponds with speech, such as a narrator's voice, currently heard in the TV show. 


\section{REFERENCES}

1. Edward Anstead, Steve Benford, and Robert J. Houghton. 2014. Many-screen viewing: evaluating an olympics companion application. In Proceedings of the 2014 ACM international conference on Interactive experiences for TV and online video (TVX '14), 103110. http://dx.doi.org/10.1145/2602299.2602304

2. Santosh Basapur, Gunnar Harboe, Hiren Mandalia, Ashley Novak, Van Vuong, and Crysta Metcalf. 2011. Field trial of a dual device user experience for iTV. In Proceedings of the 9th international interactive conference on Interactive television (EuroITV'11), 127-136. http://dx.doi.org/10.1145/2000119.2000145

3. Andy Brown, Michael Evans, Caroline Jay, Maxine Glancy, Rhianne Jones, and Simon Harper. 2014. HCI over multiple screens. In Extended Abstracts on Human Factors in Computing Systems (CHI EA '14), 665-674. http://dx.doi.org/10.1145/2559206.2578869

4. Pablo Cesar, Dick CA Bulterman, and A. J. Jansen. 2008. Usages of the secondary screen in an interactive television environment: Control, enrich, share, and transfer television content. In Proceedings of European Conference on Interactive Television, pp. 168-177. http://doi.org/10.1007/978-3-540-69478-6_22

5. Leon Cruickshank, Emmanuel Tsekleves, Roger Whitham, Annette Hill, and Kaoruko Kondo. 2007. Making interactive TV easier to use: Interface design for a second screen approach. The Design Journal 10, 3: 41-53. http://doi.org/10.1007/s00779-015-0867-7

6. John Dowell, Sylvain Malacria, Hana Kim, Edward Anstead. 2015. Companion apps for information-rich television programmes: representation and interaction. Personal Ubiquitous Comput. 19, 7: 12151228. https://doi.org/10.1007/s00779-015-0867-7

7. David Geerts, Pablo Cesar, and Dick Bulterman. 2008. The implications of program genres for the design of social television systems. In Proceedings of the 1st international conference on Designing interactive user experiences for $T V$ and video (UXTV'08), 71-80. http://dx.doi.org/10.1145/1453805.1453822

8. Michael E. Holmes, Sheree Josephson, and Ryan E. Carney. 2012. Visual attention to television programs with a second-screen application. In Proceedings of the Symposium on Eye Tracking Research and Applications (ETRA '12), 397-400. http://dx.doi.org/10.1145/2168556.2168646
9. Gaby Hornsby (producer), 2012, Seven ages of starlight. Television Broadcast. BBC: London. http://www.bbc.co.uk/programmes/p00yb434

10. Theo Jones, 2011. Designing for second screens: The autumnwatch companion. BBC, London.

http://www.bbc.co.uk/blogs/legacy/researchanddevelop ment/2011/04/the-autumnwatch-companion---de.shtml

11. Jeremy Klein, Jonathan Freeman, David Harding, and Ali Teffahi. 2014. Assessing the impact of second screen. OFCOM, London.

https://www.ofcom.org.uk/_data/assets/pdf_file/0022/

71653/second_screens_final_report.pdf

12. Lin Lin, Jennifer Lee and Tip Robertson. 2011. Reading while watching video: The effect of video content on reading comprehension and media multitasking ability. Journal of Educational Computing Research 45, 183-201.

13. Abhishek Nandakumar and Janet Murray. 2014. Companion apps for long arc TV series: supporting new viewers in complex storyworlds with tightly synchronized context-sensitive annotations. In Proceedings of the 2014 ACM international conference on Interactive experiences for TV and online video (TVX '14), 3-10. http://dx.doi.org/10.1145/2602299.2602317

14. Timothy Neate, Michael Evans, and Matt Jones. 2016. Designing Visual Complexity for Dual-screen Media. In Proceedings of the $2016 \mathrm{CHI}$ Conference on Human Factors in Computing Systems (CHI '16), 475-486. http://dx.doi.org/10.1145/2858036.2858112

15. Anna Van Cauwenberge, Gabi Schaap and Rob Van Roy. 2014. TV no longer commands our full attention: Effects of second-screen viewing and task relevance on cognitive load and learning from news. Computers in Human Behavior 38: 100-109. http://doi.org/10.1016/j.chb.2014.05.021 